\title{
CINCO TEORÍAS SOBRE EL CONCEPTO DE LOS DERECHOS *
}

\author{
Antonio Manuel Peña Freire \\ Universidad de Granada
}

RESUMEN. En este artículo se analizan cinco teorías sobre el concepto de los derechos: la teoría de la voluntad, la teoría del interés y otras tres, más recientes, que han revitalizado el debate sobre este asunto, como son la teoría de la restricción justificada de G. RAINBOLT, la teoría de la diversidad de funciones de L. WenAR y la teoría híbrida de G. SREENIVASAN. Las tres primeras son consideradas simples por presuponer que los derechos tienen una referencia homogénea y que, por lo tanto, es posible un concepto único para todos los casos de derechos. Las dos últimas, por los motivos opuestos, son complejas. Finalmente se formulan algunas consideraciones relativas a la proliferación de los derechos y también a la dimensión histórica del fenómeno que favorecerían a las teorías complejas respecto de sus rivales simples.

Palabras clave: teoría de la voluntad, teoría del interés, teoría de la restricción justificada, teoría de la diversidad de funciones, G. Rainbolt, L. WEnAR, G. SREEnivasan.

ABSTRACT. This article analyzes five theories that deal with the concept of rights: the Will Theory, the Interest Theory and three more recent theories which have revitalized the debate on this matter, G. RaInBolt's Justified Constraint Theory, L. WenAR's Several Function Theory and G. SREENIVASAN'S Hybrid Theory. The first three ones are considered to be simple for presupposing that rights have a homogeneous reference and, therefore, a unique concept is possible for all the cases of rights. The last ones, for the opposite reasons, are complex. Finally I set out some considerations about the proliferation of rights and also about the historical dimension of the phenomenon that favour a complex approach instead of a simple one.

Keywords: the Will Theory, the Interest Theory, the Justified Constraint Theory, the Several Function Theory, G. Rainbolt, L. WenAR, G. SREENIVASAN.

* Fecha de recepción: 28 de septiembre de 2009. Fecha de aceptación: 29 de octubre de 2009. 


\section{PRELIMINARES}

En este artículo se confrontan ciertas teorías que compiten entre sí por definir el concepto de los derechos, quizás uno de los más complejos y escurridizos de los que manejan teóricos y filósofos del Derecho. Un elemento común a muchos de los promotores y partidarios de esas teorías es que adoptan como punto de partida el análisis desarrollado a principios del siglo pasado por W. N. HoHFELD. Por este motivo, será necesaria una referencia sucinta a las tesis de este autor antes de entrar propiamente en materia. Es sabido que HOHFELD, preocupado por la ambigüedad de expresiones del tipo de «tener un derecho» y por la desmesura en el recurso a las nociones de derecho y deber ${ }^{1}$, propuso considerar la singularidad de cuatro relaciones jurídicas fundamentales, formadas, cada una de ellas por una pareja de posiciones jurídicas. En el siguiente cuadro aparecen las citadas relaciones y, de arriba abajo, sus respectivas posiciones integrantes:

\begin{tabular}{|c|c|c|c|}
\hline 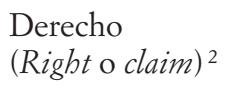 & $\begin{array}{l}\text { Privilegio o libertad } \\
\text { (Privilege o liberty) }^{3}\end{array}$ & $\begin{array}{l}\text { Potestad } \\
\text { (Power) }\end{array}$ & $\begin{array}{l}\text { Inmunidad } \\
\text { (Immunity) }\end{array}$ \\
\hline $\begin{array}{l}\text { Deber } \\
\text { (Duty) }\end{array}$ & $\begin{array}{l}\text { No-derecho } \\
(\text { No-right })\end{array}$ & $\begin{array}{l}\text { Sujeción } \\
\text { (Liability) }\end{array}$ & $\begin{array}{l}\text { Incompetencia } \\
\text { (Disability) }\end{array}$ \\
\hline
\end{tabular}

HOHFELD fue parco a la hora de definir cada una de estas relaciones pues entendía que, al ser fundamentales, cualquier definición formal sería insatisfactoria si no estéril. En su lugar, prefirió ejemplificarlas y, a continuación, vincularlas mediante relaciones de correlación y oposición ${ }^{4}$. Las definiciones resultantes son las siguientes:

1. Derecho/deber. La definición precisa de lo que sea un derecho en sentido estricto se sostiene sobre su correlativo: el deber. A tiene un derecho frente a B cuando B tiene un deber hacia o para A de hacer o no hacer algo; si el deber se incumple, enton-

\footnotetext{
1 «One of the greatest hindrances to the clear understanding, the incisive statement and the true solution of legal problems frequently arises from the express or tacit assumption that all legal relations may be reduced to "rights" and duties» (HOHFELD, 1919, p. 35).

2 Aunque HoHFeLD empleó «right» en sus escritos, el término con el que normalmente se designa a esta posición en inglés es «claim». La traducción al castellano de «claim» («a demand for something due or believed to be due» según el MERRIAM-WEBSTER) es complicada, pues traducido como «Derecho» se solapa con la traducción de la matriz right al que claim pretende aclarar. A. Ross (1958, p. 152), en otro de los textos de referencia sobre este tema, emplea «claim», que es traducido por G. CARRIó al español como «facultad», lo que no parece muy acertado. El propio CARRIó, al traducir a HOHFELD, usará el término «derecho» para traducir el «right» hohfeldiano. Quizás fuese posible traducir «claim» como «reclamación» o «reivindicación», lo que solventa la ambigüedad aunque parecen lo suficientemente generales en español. Se ha optado por traducir claim como «Derecho» e incluir mención al original entre paréntesis o la indicación de «en sentido estricto» —en esto sigo el criterio de CARRIÓ, 1968, p. 14- cuando el contexto no permitiese evitar la confusión con la noción más genérica de right.

3 HOHFELD empleó el término «privilege», que CARRIÓ (1968, p. 14) traduce como «privilegio», aunque posteriormente se ha generalizado el de «liberty» para denominar esta posición. Ross (1958, p. 158) emplea ya el término «liberty», traducido convenientemente al castellano como «libertad». Se ha seguido este criterio.

${ }^{4}$ En el cuadro serían correlativas las posiciones en la vertical —esto es, derecho es correlativo de deber, libertad de no-derecho, potestad de sujeción e inmunidad de incompetencia- y opuestas las posiciones de derecho con no-derecho, libertad con deber, potestad con incompetencia e inmunidad con la posición de sujeción.
} 
ces diremos que el derecho ha sido violado (HoHFELD, 1919, pp. 36-38). La acción u omisión a la que A tiene derecho coincide con la acción u omisión debida por B. Esta acción u omisión es el contenido de la relación, A es el sujeto activo del derecho y B, en tanto que titular del deber correlativo, es su sujeto pasivo ${ }^{5}$.

2. Libertad/no-derecho. Una libertad es, en el esquema hohfeldiano, lo opuesto a un deber y lo correlativo a un no-derecho. A tiene una libertad para hacer una acción $\mathrm{x}$ cuando no tiene el deber de no hacer $\mathrm{x}$ frente a otro sujeto B (HOHFELD, 1919, pp. 3850). Una libertad para hacer algo es, por tanto, lo contrario a un deber de contenido opuesto, es decir, referido a la conducta opuesta a la que es contenido de la libertad ${ }^{6}$ : se es libre para hacer algo cuando no se tiene el deber de no hacerlo y se es libre para no hacer si no se tiene el deber de hacer ${ }^{7}$. La posición correlativa de una libertad es un no-derecho: así el correlativo de la libertad de $\mathrm{A}$ para fumar es el no-derecho de $\mathrm{B}$ a que $\mathrm{A}$ no fume o, dicho de modo más natural, que $\mathrm{B}$ no tiene derecho a que $\mathrm{A}$ no fume, lo que, a su vez, equivale a negar un deber de A hacia B de fumar. HoHFELD (1919, p. 39) reconoce que es común confundir derecho y libertad aunque para él eran dos relaciones distintas ya que una libertad podría existir sin el derecho a no ser interferido en aquello que para lo que uno es libre. Veamos un caso: A puede ser libre de hacer algo —en el ejemplo de HoHFELD (1919, p. 41) comer una ensalada - frente a otros, digamos $\mathrm{B}, \mathrm{C}$ y $\mathrm{D}$, si al comer la ensalada no viola el derecho de $\mathrm{B}, \mathrm{C}$ o $\mathrm{D}$ a que $\mathrm{A}$ no la coma: efectivamente, si $\mathrm{B}, \mathrm{C}$ y $\mathrm{D}$ no tienen derecho a que $\mathrm{A}$ no coma la ensalada, entonces no hay deber para A de no comerla o, lo que es lo mismo, A es libre para hacerlo. Sin embargo, que A sea libre para comer la ensalada frente a B, C y D no significa que $\mathrm{B}, \mathrm{C}$ o $\mathrm{D}$ tengan algún tipo de deber hacia $\mathrm{A}$ de abstenerse de interferir con A o de no impedirle a A comerla. Si B, C y D tuvieran el deber de no interferir, entonces A tendría un derecho frente a B, C y D. Como A sólo tiene una libertad, si, por ejemplo, $\mathrm{B}$ es más rápido que $\mathrm{A}$, coge antes el plato de ensalada y lo come, $\mathrm{B}$ no habrá violado derecho alguno de A ni, por supuesto, su libertad para comer la ensalada, pues la negación del deber de comerla no habilita a comerla ni otorga ventaja alguna al respecto. En suma, el contenido de una libertad y el de un derecho son distintos: cuando decimos no tener el deber de hacer algo estamos ante una libertad y cuando otros tienen el deber de no interferir con nuestra acción, entonces tenemos un derecho frente a ellos ${ }^{8}$.

5 A veces se le denomina también «objeto», por ejemplo, RAINBOLT, 2006, p. 1.

6 KRAMER (1998, p. 10), con precisión, señala que tener una libertad para llevar a cabo cierta acción implica estar libre de cualquier deber de no llevarla a cabo, mientras que tener la libertad de abstenerse de llevar a cabo esa acción implica la libertad frente a cualquier deber de ejecutarla o realizarla. La libertad para hacer x se opone, no al deber de hacer x, sino al deber de no hacerlo y correlativamente, lo contrario de la libertad para no hacer x sería el deber de hacerlo (KRAMER, 1998, p. 13).

7 HoHFELD (1919, p. 39) apostilla que ser libre para hacer — es decir, no tener el deber de no hacer xes compatible con tener el deber de hacer. Es más, parece que, en un sistema coherente, ambas posiciones se requieren pues de no concurrir estaríamos en presencia de una antinomia ya que si se tiene el deber de hacer y no es cierto que se tenga el deber de no hacer, entonces simultáneamente se tiene el deber de hacer y el deber de no hacer. Un análisis en clave lógica de esta doble posibilidad en HALPIN (2003, pp. 41 y ss., esp. 46 y ss.).

8 KRAMER (1998, pp. 15-16) se refiere incluso a la existencia de derechos sin libertades en el caso de que alguien tenga derecho a que no se le impida hacer algo que, sin embargo, no tiene permiso para hacer. En concreto ofrece un ejemplo que alude al derecho del dueño de una fábrica situada en un lago a que los ribereños no le impidan realizar unos vertidos que debería no realizar. En el esquema hohfeldiano, por tener el deber de no verter, diríamos que no es libre para verter, pero por tener los demás el deber de no impedir el vertido, diremos que el industrial tiene un derecho a no ser interferido en caso de que decidiera llevar a cabo el vertido. 
3. Potestad/sujeción. Excepcionalmente, en el caso de la definición de las potestades encontramos en HOHFELD referencias a un rasgo, en apariencia más básico, a partir del que son caracterizadas. HoHFeld (1919, p. 51) define la potestad como la habilidad legal de uno o varios seres humanos para provocar ciertos cambios normativos a partir de determinados actos que están bajo el control de su voluntad. Es titular de la potestad el sujeto cuyos actos dan lugar a cambios normativos en otras relaciones jurídicas y están sujetos a la potestad los titulares de las relaciones jurídicas alteradas como consecuencia de su ejercicio, es decir, aquellos cuyas relaciones se ven deliberadamente alteradas a consecuencia de la realización del denominado acto desencadenante (triggering act $)^{9}$. En este punto, también es importante distinguir entre la potestad como poder para dar lugar a cambios normativos y la libertad de ejercerla (HOHFELD, 1919, p. 58): uno puede ser hábil para provocar ciertos cambios normativos y, por tanto, tener potestad, y, sin embargo, puede tener el deber de no ejercerla, es decir, que puede no ser libre para ejercerla ${ }^{10}$.

4. Inmunidad/incompetencia. Respecto de la inmunidad, HoHFELD (1919, p. 60) establece que la inmunidad es lo contrario de la sujeción y tiene por correlativa a una incompetencia, que a su vez, es lo contrario de la potestad. HOHFELD concluye la caracterización de esta relación trazando un ilustrador paralelismo de la relación existente entre potestades e inmunidades y la que se da entre derechos y libertades: un derecho permite afirmar una demanda o exigencia frente a otro y una libertad es la exención respecto de las exigencias de otro; la potestad, por su parte, permite la afirmación o el control sobre las relaciones normativas de otro, mientras que la inmunidad es, en algún modo, la libertad frente a la potestad de otro respecto de cierta o ciertas relaciones jurídicas ${ }^{11}$.

\section{TEORÍAS SOBRE LOS DERECHOS}

Es habitual considerar al esquema hohfeldiano como la quintaesencia del concepto de los derechos y, sin embargo, en mi opinión, aun siendo un buen punto de partida, es un error pensar que agota todos los aspectos o problemas que la definición de los dere-

\footnotetext{
Añadiré yo que aunque los respectivos contenidos de ambas relaciones no coinciden, el ejemplo es iluminador e incluso podríamos pensar en ejemplos similares en el caso del propietario de una vivienda arrendada frente a los inquilinos que han dejado de pagar la renta: éstos, por tener el deber de marcharse, no son libres de permanecer en la casa y, sin embargo, tienen derecho a no ser expulsados hasta que se resuelva el juicio de desahucio y el propietario tiene, también hasta ese momento, el correlativo deber de no interferir ni perturbar en el disfrute de la vivienda.

9 Así podríamos denominar al acto que, por dar lugar a los cambios, implica el ejercicio de la potestad. $\mathrm{Al}$ respecto véase RAINBOLT, 2006, pp. 10-17.

10 Por último, y pese a que los escritos de HoHFELD sobre este punto son algo confusos, en el caso de relación potestad/sujeción, el sujeto pasivo y activo no tienen por qué ser distintos. Según KRAMER (1998, p. 20) uno tiene un poder o potestad cuando puede expandir, reducir o, en algún sentido, modificar sus propios títulos o los títulos de otra persona, con lo que es posible que uno sea simultáneamente titular y sujeto de la potestad.

${ }_{11}$ Como ocurría en el caso de la relación potestad/sujeción, hay que notar también que uno puede ser inmune frente a sí mismo, es decir, que un mismo individuo puede ser simultáneamente sujeto pasivo y activo de la inmunidad. Uno, por ejemplo, no puede venderse como esclavo ni tampoco acordar una prestación laboral con una jornada superior a ciertos máximos o una remuneración inferior a ciertos mínimos. En este sentido, uno tiene una inmunidad frente a actos propios, es decir, que es incompetente para alterar en esos aspectos su propio estatuto normativo.
} 
chos suscita. No es posible encontrar en HOHFELD respuestas a todos los interrogantes que el concepto de los derechos plantea puesto que el cuadro de relaciones jurídicas fundamentales es, a la vez, algo más y algo menos que una definición de los derechos ${ }^{12}$. Es algo más que un concepto de los derechos pues es más general ya que se refiere a las relaciones jurídicas que pueden plantearse entre dos sujetos o partes; algunas de estas relaciones podrían ser consideradas casos típicos de derechos, pero otras no. Como señaló CARRIÓ (1968, p. 7), el cuadro hohfeldiano se encontraría a medio camino entre las nociones o expresiones que sirven para describir el estatuto normativo de las acciones de un sujeto ${ }^{13} \mathrm{y}$, de otro lado, instituciones o entramados normativos mucho más complejos, característicos de sectores específicos de los ordenamientos jurídicos, como, por ejemplo, las nociones de contrato, herencia, aval, demanda o, por supuesto, de tal o cual derecho tal y como lo podemos encontrar en una constitución típica o en un texto legal real cualquiera. Las relaciones que HOHFELD analiza son, así, menos complejas que el derecho a cobrar una deuda, la libertad de expresión, la patria potestad, la potestad legislativa o la inmunidad diplomática, aunque, desde luego, también son imprescindibles en el análisis de todas esas relaciones.

En segundo lugar, dije que el análisis hohfeldiano es algo menos que un concepto de los derechos porque la respuesta a la pregunta por lo que sea un derecho o cuáles relaciones son derechos no puede encontrarse sólo en el mentado cuadro ${ }^{14}$ y requiere la consideración de factores o rasgos adicionales a la reproducción de una estructura básica. Tradicionalmente dos teorías han intentado dar respuesta a esta cuestión: la teoría de la voluntad y la teoría del interés. Ambas afirman que un rasgo - la voluntad o el interés- es definitorio de las relaciones jurídicas a las que se puede considerar como casos de derechos. Por presuponer que existe un único rasgo que define a todos los derechos, es decir, por presuponer una homogeneidad interna en la referencia del concepto de los derechos, las consideraré teorías simples de los derechos. En el siguiente epígrafe analizaré someramente ambos planteamientos teóricos dado que su confrontación es un lugar común en los estudios sobre los derechos y también porque son introductorias de otras tres teorías a las que me referiré con un mayor detenimiento.

Efectivamente el debate entre teóricos de los derechos partidarios de la voluntad o del interés parecía encontrarse hasta hace poco en una situación de bloqueo y, sin embargo, se ha visto revitalizado por tres nuevos planteamientos que se presentan a sí mismos como formas de superar la dialéctica entre voluntad e intereses. La primera propuesta innovadora - aunque es simple como las dos anteriores- es la teoría de la restricción justificada de G. RAINBOLT. Tras presentar sus tesis básicas sobre el concepto de los derechos, formularé algunas objeciones y consideraciones críticas. Terminaré exponiendo los rasgos básicos de la teoría de la diversidad de funciones de los derechos de L. WENAR

12 Que los trabajos de HOHFELD hagan referencia a «relaciones jurídicas fundamentales» es bastante significativo y autorizaría la conclusión de que su genuino objeto de estudio eran las relaciones jurídicas en general y no los derechos en particular. Las referencias a la imprecisión o ambigüedad de los términos y expresiones con las que designamos a los derechos podrían ser puramente incidentales o ejemplificativas en ese propósito último.

13 También Hierro (2000, p. 156), en un extenso y documentado estudio, adopta una postura similar al distinguir entre modalidades deónticas y conceptos jurídicos básicos.

${ }_{14}$ Como dice con claridad ELEFTHERIADIS (2008, p. 115) si un derecho es un deber hacia alguien, la definición de los derechos no estará completa hasta que no se determine qué diferencia hay entre un deber a secas y un deber hacia alguien. Conclusión similar la de WALDRON (1984, p. 8). 
y de la teoría híbrida de G. SREENIVASAN. Estas teorías son innovadoras, como la anterior, pero, como veremos, son complejas, porque - a diferencia de las teorías de la voluntad, del interés y de la restricción justificada— no ven posible una definición única para todos y cada uno de los derechos y consideran que existen categorías específicas de derechos con rasgos propios que podrían no presentarse en otros de otras categorías.

\section{TEORÍAS SIMPLES DE LOS DERECHOS}

\subsection{La teoría de la voluntad}

La teoría de la voluntad considera que los derechos son instrumentos para la expresión de la voluntad y de las decisiones de los individuos. La libertad, por su parte, es la razón justificatoria de los derechos. No es casual que la primera manifestación de la teoría de la voluntad se localice en la filosofía de KANT para quien los derechos equivalen a la posesión del arbitrio de otro o, dicho de otro modo, a la capacidad de uno para determinar la conducta de otro conforme a una ley universal de libertad. Esta formulación primigenia luego inspiraría a autores como SAVIGNY o WINDSCHEID quienes definieron los derechos como un ámbito donde la voluntad de su titular impera si bien condicionada por las reglas jurídicas, o como poderes de la voluntad reconocidos jurídicamente ${ }^{15}$. En el ámbito anglosajón H. L. A. HART es el referente básico de la teoría de la voluntad. Para HART (1982) el titular de los derechos es un soberano a escala (HART, 1982, p. 183) y los derechos son elecciones protegidas (protected choices), esto es, relaciones entre dos sujetos donde uno es libre para hacer o no hacer algo y el otro tiene ciertas obligaciones o deberes dirigidos a evitar su interferencia en la acción del primero. HART (1982, pp. 188 y ss.) compone la estructura típica de los derechos uniendo dos libertades hohfeldianas con un deber de no interferencia, es decir, que un derecho equivale a una libertad bilateral, que permite llevar a cabo o no una acción propia, generalmente de tipo natural o bruto ${ }^{16}$, más un deber anexo de no interferir o de tolerar esa alternativa de acción. Junto a este tipo básico de derechos, hay otros cuyo contenido se refiere a un acto al que el ordenamiento jurídico atribuye una significación especial, como por ejemplo, contratar, testar, votar, etcétera. Estos derechos, nos dice HART, son equivalentes a potestades. Una tercera categoría son los derechos sobre deberes de otros que son, para HART, una especie de potestades, ya que el derecho aquí equivale a la potestad para controlar el deber correlativo, en el sentido de que se podría cancelarlo, reclamarlo judicialmente y exigir la reparación que corresponda por el incumplimiento o bien dejar de hacer todas esas cosas.

Han sido muchas las objeciones planteadas a la teoría de la voluntad. La existencia de derechos sin voluntad alguna que los respalde ${ }^{17}$ o la titularidad de derechos por

15 Una síntesis de las tesis de la pandectística alemana en DABIN (1952, pp. 70-81). Una exposición de esas tesis combinada con las manifestaciones características del ámbito anglosajón en ELEFTHERIADIS (2008, pp. 8 y ss.). Un interesante análisis de la dimensión moral o política de la teoría de la voluntad en SIMMONDS (1998, pp. 134-145).

16 En el ejemplo de HART, mirar por encima de una cerca; vale también desplazarse, expresarse, etcétera.

17 Vid., al respecto, DABIN (1952, pp. 75-76). 
parte de personas incapaces para expresar su voluntad ${ }^{18}$ y la existencia de derechos indisponibles o irrenunciables a voluntad de su titular ${ }^{19}$, son algunas de ellas. Una objeción de mayor calado teórico apuntaría a la parcialidad de la teoría de la voluntad dada su vinculación ideológica con la filosofía política liberal y con cierta concepción defensiva o negativa de los derechos y de la relación entre los individuos y los poderes públicos ${ }^{20}$ : los teóricos de la voluntad — se afirma- no estarían en modo alguno describiendo analíticamente el concepto de los derechos sino que lo postularían de un modo parcial, condicionado por su compromiso político con la libertad y la autonomía moral. Adicionalmente se advierte que la teoría se habría visto desbordada por la evolución social, política y jurídica, con lo que una concepción liberal de los derechos habría quedado superada por los acontecimientos y sería, si acaso, una teoría válida en algún momento pasado pero no en la actualidad ${ }^{21}$.

\subsection{Las teorías del interés}

La alternativa a las teorías de la voluntad son las teorías del interés que conciben a los derechos como instrumentos para promover y proteger el bienestar y los intereses de los individuos. Un sujeto, por tanto, tendría un derecho si otro tiene el deber de llevar a cabo cierto acto en interés del primero, es decir, que lo determinante a la hora identificar o de atribuir derechos no es la voluntad de nadie, sino el provecho o el beneficio que alguien obtiene del cumplimiento de una obligación.

En los orígenes de las teorías del interés encontramos las tesis de BENTHAM a propósito de la relación entre derechos y beneficiarios de obligaciones ${ }^{22}$. En el modelo de BENTHAM ser titular de un derecho significa ser el beneficiario de una obligación de otro $^{23}$. Junto a esta versión de la teoría del interés, denominada protectora por poner el énfasis en la función protectora o de preservación que los derechos ejercen sobre los intereses de los sujetos, existe otra variante de la teoría del interés denominada justificatoria ${ }^{24}$. Un individuo, según esta versión, tendría un derecho cuando un interés o algún aspecto de su bienestar es razón suficiente para sostener que otro sujeto o sujetos tienen un deber, es decir, que afirmar que alguien tiene un derecho supone sostener que un aspecto del bienestar de esta persona es una razón que requiere un cierto comportamiento en otros (RAZ, 1986, p. 180). Los derechos, por tanto, son los fundamentos de los deberes de otros (RAZ, 1986, pp. 166-167) ${ }^{25}$.

18 KRAMER (1998, pp. 69 y ss.) o MACCORMiCK (1976, pp. 305 y ss.).

19 Maccormick (2008, p. 327) o Rainbolt (2006, p. 102).

20 WENAR $(2005$, p. 203).

21 SPECTOR (2007, p. 292).

22 La referencia a R. VON IHERING es también ineludible. Una síntesis de la teoría del interés de IHERING en DABIN (1952, pp. 81-90)

23 Bentham (1782, p. 58) citado por Eleftheriadis (2008, pp. 8-9) y HART (1982, pp. 196-198).

24 Un contraste entre ambas versiones en RAINBOLT (2006, p. 86).

25 Entre nosotros LAPORTA (1987, pp. 27, 29), tras considerar un error la identificación de los derechos con las técnicas de protección que se les dispensan, suscribe una versión justificatoria de la teoría del interés ya que, afirma, la noción de los derechos es inseparable de la dimensión del «bien», «satisfacción de interés»o «beneficio» para su titular, de modo que un derecho sería la razón o título expresivo de una especial consideración que los sistemas normativos atribuyen a ciertas situaciones o estados de cosas relativos a los individuos de una cierta clase, y que justifica la adscripción y la protección de ese bien. 
La teoría del interés también ha sido objeto de diversas objeciones. La más inmediata sugiere que existen derechos que no benefician a su titular ni son de su interés por el simple motivo de que podrían ir en su perjuicio ${ }^{26}$. Otra objeción común apunta a la existencia de terceros beneficiarios o beneficiarios en cascada del cumplimiento de ciertos deberes, quienes, de acuerdo a la teoría del interés, tendrían que considerarse titulares de un derecho frente a un obligado con el que podrían no tener vínculo relevante alguno ${ }^{27}$. También se ha criticado la falta de correspondencia que existe en muchas ocasiones entre el peso del interés protegido o justificatorio y el peso del derecho $^{28}$. Esa dimensión del peso abriría además la puerta al cálculo utilitario en materia de derechos y permitiría la comparación y ponderación así como las compensaciones o ajustes entre derechos e intereses concurrentes en un caso. Como consecuencia, la teoría del interés no podría dar cuenta del hecho de que los derechos son concebidos con frecuencia como factores o razones que están fuera de cualquier cálculo utilitario ${ }^{29}$ y que, por tanto, no pueden ser confrontados con otros intereses del sujeto titular o con intereses generales ${ }^{30}$. Por último, y como ocurría con la teoría de la voluntad, se ha destacado la vinculación de la teoría del interés a filosofías y planteamientos políticos welfaristas ${ }^{31}$, lo que también la convertiría en una tesis ideológicamente condicionada y parcial en el seno de un debate pretendidamente analítico.

\subsection{La teoría de la restricción justificada}

La teoría de la restricción justificada (Justified-Constraint Theory), propuesta por G. RAINBOLT (2006), no identifica el rasgo definitorio de los derechos en algún rasgo $\mathrm{o}$ atributo de su titular, sino en la restricción que suponen para el comportamiento de otros sujetos distintos a su titular, es decir, que hace pivotar su concepto de los derechos sobre el sujeto pasivo de los mismos, esto es, sobre el que — dicho en términos

${ }^{26}$ Los teóricos del interés han intentado sortear esta objeción distinguiendo entre el beneficio en general y, de otro lado, los casos particulares de perjuicio que no serían teóricamente relevantes. Vid. RAz (1986, p. 168), MACCORMICK (2008, p. 329) o KRAMER (1998, pp. 93-97).

27 KRAMER (1998, pp. 79-84) elabora una solución a esta objeción; SREENIVASAN (2005, p. 264) considera que, pese a todo, el problema persiste.

${ }_{28}^{28}$ Una exposición de la objeción y una posible solución en RAZ (2008, p. 347).

29 Así ocurre, por ejemplo, cuando primamos la capacidad de elegir y no el carácter benéfico o perjudicial de aquello que finalmente se elige, o cuando definimos los derechos como triunfos o títulos frente a consideraciones atinentes al interés general o social.

30 SPECTOR $(2007$, p. 293$)$.

31 Para ElefTHERIADIS (2008, p. 15), la teoría del interés, como ocurría con la de la voluntad, termina siendo no descriptiva o explicativa de su objeto, como corresponde a una teoría de corte analítico, sino distorsionadora del mismo ante la necesidad de explicarlo en función de unos patrones normativos previamente aceptados. KRAMER $(1998$, p. 79) rechaza estas críticas y señala que la teoría del interés no es necesariamente normativa, pues simplemente define la estructura de los derechos a partir de la idea del interés protegido o cualificado, sin decir qué intereses tendrían que serlo, tarea esta que correspondería a la filosofía política o al discurso político ordinario. La teoría de la voluntad, sin embargo, por su vinculación inmediata con el valor de la autonomía personal, sí estaría indisolublemente conectada a ciertas asunciones y preferencias normativas y, por tanto, sería vulnerable a la crítica que subraya su parcialidad. No obstante, estas consideraciones de KRAMER podrían ser refutadas si pensamos que el mero hecho de admitir como normal la posibilidad de definir los derechos en términos completamente independientes a la voluntad de sus titulares o de construir catálogos de derechos en los que no hubiese ningún elemento que implicase la protección o reconocimiento de la autonomía personal, es ya un planteamiento normativo claro que deja traslucir posicionamientos ideológicos precisos. 
hohfeldianos - se encuentra en la posición correlativa a la del titular del derecho. El punto de arranque para la reconstrucción del concepto de los derechos, por tanto, se encuentra en la idea de constreñimiento de los actos de un tercero.

RAINBOLT asume - con matizaciones - el instrumental metodológico hohfeldiano y añade que las únicas relaciones o posiciones que implican restricciones normativas sobre otros son los derechos en sentido estricto (claims) y las inmunidades (RAINBOLT, 2006, p. 26) ${ }^{32}$. Si uno tiene un deber de hacer algo estará constreñido en el sentido de que están limitadas las acciones normativamente permitidas para ese sujeto; del mismo modo, en el caso de la inmunidad se considerará constreñido al sujeto incompetente en tanto que no puede decidir sobre cierta cuestión ni alterar determinadas relaciones o posiciones normativas del sujeto inmune. Derechos (claims) e inmunidades son las únicas relaciones hohfeldianas cuya titularidad implica una restricción normativa sobre otro, de ahí que sean las únicas que pueden ser consideradas, en sí mismas, derechos; no obstante, cualquier otra relación, siempre que esté protegida por un derecho (claim) o una inmunidad, podrá ser también considerada un caso de Derecho ${ }^{33}$.

Ahora bien, derecho no es sin más equivalente al correlativo de una restricción, de modo que no todas las restricciones pueden ser consideradas sin más generadoras de derechos. RainBOlT (2006, pp. 119-120) añade que la restricción que suponen los derechos es debida a algún rasgo o propiedad de una persona y no del mundo. Los derechos de un sujeto son los correlativos a los deberes y las incompetencias de otros que se justifican en algún rasgo o propiedad del sujeto titular y no del mundo. En su formulación canónica, la teoría de la restricción justificada afirmaría que

«X tiene un derecho $\mathrm{S}$ frente a $\mathrm{Y}$ a que $\mathrm{Y}$ haga $\mathrm{A}$ si y sólo si es el caso de un argumento sólido y no superfluo con la siguiente forma:

1. $\mathrm{X}$ es un $\mathrm{F}$

2. si $\mathrm{X}$ es un $\mathrm{F}$, entonces $\mathrm{Y}$ tiene la obligación/imposibilidad $\mathrm{S}$ de hacer $\mathrm{A}$

3. Luego $\mathrm{Y}$ tiene la obligación/imposibilidad $\mathrm{S}$ de hacer $\mathrm{A}$ ».

El carácter último de esa propiedad o rasgo personal no está muy claro en los escritos de RAINBOLT ${ }^{34}$, pues pese a referirse al mismo como la razón de la restricción, lo cierto es que el sentido de la teoría no apunta a consideraciones normativas que fundamenten el derecho o su fuerza vinculante, sino a rasgos o propiedades (features) del sujeto determinantes en la medida en que son el elemento fáctico que da la clave de la fundamentación de la restricción, esto es, el dato fáctico que permite comprender y dar por fundamentado el deber o la incompetencia de otro.

Lo hasta aquí resumido es suficiente para abordar el contraste de esta teoría con las otras concurrentes, así como su mayor o menor éxito a la hora de dar cuenta adecuadamente a algunos de los retos planteados por el concepto de los derechos. La teoría de RAINBOLT tiene diversos méritos incuestionables en su haber: solventa los problemas extensionales que planteaban las teorías de la voluntad y del interés, pues su definición abarca los casos de derechos que aquellas dos teorías no incluían o incluían con dificultad. También es interesante el hecho de que preste particular atención a la idea de

\footnotetext{
32 Respalda esta tesis citando a STEINER (1994, pp. 55-59).

33 Una solución análoga, aunque con matices, la de HAYWARD (2006, p. 14).

34 Algunas consideraciones adicionales sobre esta propiedad en RAINBOLT (2006, pp. 243-244).
} 
restricción en el comportamiento de otros que parece, en efecto, el modo básico en que los derechos se expresan normativamente. De un modo singular, esta proyección de los derechos me parece una faceta ineludible para la teoría jurídica que evitaría así lecturas atributistas de los derechos excesivamente volcadas sobre el sujeto titular, al poner al mismo nivel de éste, la proyección que los derechos tienen sobre otros.

Sin embargo, creo que la teoría tiene diversos puntos débiles que afectan fundamentalmente al modo en que se definen los rasgos personales fundantes de los derechos. Para exponer esta objeción contrastaré la definición de los derechos de la teoría de la restricción justificada con la de la teoría del interés en su versión justificatoria, con la que, a mi juicio, aquella finalmente se confunde. Para esa segunda teoría, cuando un interés de un sujeto es la razón que fundamenta el deber de otro, estamos en presencia de un derecho del primero. Por su parte, la teoría de la restricción justificada afirma que, no ya un interés, sino cualquier propiedad de una persona y no del mundo es hábil para fundar un derecho cuando es efectivamente la razón de una restricción de otro. Es evidente que la tesis de RAINBOLT supone una ampliación del espectro de la definición de la teoría del interés en versión justificatoria, pero, como veremos, esta ampliación termina por desbordar cualquier extensión razonable del concepto de los derechos.

Intentaré formular un caso ejemplificativo de lo que quiero decir y para ello me referiré a una sociedad imaginaria dividida en dos grupos de individuos identificables por el color azul o rojo de su piel y en la que los rojos son esclavos de los azules. El deber de los rojos de trabajar para los azules a cambio de nada es una restricción normativa en el comportamiento de los rojos que autorizaría a hablar de un derecho de los azules a que los rojos trabajen para ellos. Además supongamos que los azules, en ese mundo imaginario, son incompetentes para cambiar el estatuto normativo de los rojos que, por tanto, son inmunes frente a cualquier acto de los azules que pretendiese su manumisión. Según los parámetros de la teoría de la restricción justificada, la inmunidad correlativa a esta incompetencia es un derecho porque implica una restricción normativa cuya justificación reposa necesariamente sobre un rasgo del sujeto titular - el color rojo de la piel de parte de la población- y no del mundo: así las cosas, habríamos de concluir afirmando que los rojos tienen derecho a no ser liberados de su condición de esclavos por los azules.

Opino, sin embargo, que la estructura normativa resultante está lejos de ser un caso del tipo de las relaciones comúnmente consideradas como derechos. A conclusiones parecidas se había llegado antes analizando el conocido caso de la inmunidad frente a la concesión de una pensión de jubilación a un vecino por parte de un ayuntamiento ${ }^{35}$ : esa inmunidad — simple reflejo de la incompetencia correlativa — en modo alguno parece corresponderse a lo que típicamente se piensa que es un derecho. Lo que tienen en común el color rojo de mi ejemplo y las competencias de los ayuntamien-

35 HART (1982, p. 199) y MACCORMICK (2008, p. 330). Un caso análogo fue sugerido a RAINBOLT por un lector anónimo de su libro que refirió el deber de un padre de no dejar sola a su hija con un vecino pedófilo. Si la pedofilia del vecino es el hecho que da la clave del deber, entonces el deber del padre es hacia el pedófilo que automáticamente quedaría convertido también el titular de un derecho a que el padre no deje sola a su hija con él. RaINBOLT (2006, p. 124) intenta superar la objeción recordando el carácter puramente descriptivo y formal de su teoría y señalando que la limitación de los rasgos hábiles para justificar restricciones y, por tanto, para constituir derechos, es un problema sustantivo que dependerá de consideraciones básicamente morales. 
tos en las relaciones descritas es que ambos son factores que causan ciertos constreñimientos en otros, pero que no autorizan a hablar de derechos de quienes dan lugar al constreñimiento. Los rojos dan lugar a la restricción de los azules porque son rojos, pero son los perjudicados por la restricción, igual que los vecinos son los perjudicados por el hecho de que el municipio en que residen esté constreñido en sus funciones y no pueda otorgarles una pensión a cargo del contribuyente. En ambos casos, pese a haber restricción, no hay derecho por mucho que, en términos hohfeldianos, rojos y vecinos sean titulares de una inmunidad correlativa a una incompetencia.

En una detallada respuesta privada a esta objeción RAINBOLT me recordó la necesidad de distinguir entre los derechos que podrían seguirse de una definición teórica y los derechos moralmente deseables que podrían seguirse de esa misma definición ${ }^{36}$. También comparó el caso de los rojos con el de los judíos en la Alemania nazi en lo que se refiere al deber de estos de llevar cosido a la ropa un signo identificativo de su condición, deber del que es fácil suponer que no podían ser liberados por decisión de sus vecinos arios. Respecto del caso de los rojos, cuestionó la relevancia del rasgo «rojo de piel», señalando que probablemente fuese superfluo en el proceso justificatorio de la restricción pues el hecho que realmente daría la clave de la misma sería más bien el deseo de los azules de servirse de los rojos, es decir, que lo realmente justificante y fundante del derecho serían los intereses de los azules y no el color de los rojos ${ }^{37}$.

Respecto de la necesidad de distinguir entre condiciones conceptuales y consideraciones normativas o morales, mi acuerdo con RAINBOLT no puede ser mayor. Sin embargo, el motivo porque el afirmo que casos como el «derecho a no ser liberado» de los ejemplos anteriores son absurdos no es de corte moral sino conceptual. No todas las posiciones correlativas a las restricciones de las acciones de un sujeto son derechos y no precisamente por motivos morales o no sólo por motivos morales. Una teoría que identifique un derecho de los rojos a no ser liberados por los azules es, no sólo moralmente repudiable, sino también conceptualmente errónea: no basta con ser titular de la posición correlativa a una restricción para ser titular de un derecho, sino que además esa restricción tiene que tener un determinado sentido o cumplir una cierta función desde el punto de vista del titular del derecho, que, desde luego, no está presente en casos como los anteriores.

El paralelismo histórico con los judíos en el régimen nacional-socialista refuerza — creo- mi objeción de fondo a la teoría de la restricción justificada, pues no me parece plausible presuponer ni postular un concepto de los derechos que permita afirmar que los judíos son titulares de un derecho a no ser liberados de la carga de portar el infamante signo (ya que sus vecinos arios están constreñidos en el sentido de no pueden cancelar el deber de aquéllos), por los mismos motivos por los que no

36 En su libro afirma RAINBOLT (2006, pp. 119-120) que las consideraciones morales o sustantivas son un asunto ajeno a la teoría que pretenda definir la estructura de los derechos.

37 Esta última matización plantea un problema adicional a la teoría de la restricción justificada. En el contraejemplo RAINBOLT señala como portador del rasgo que justifica la restricción al sujeto constreñido por la misma, lo que es incongruente con sus propias premisas teóricas, según las cuales los derechos son el correlativo de las restricciones de otros justificadas en algún rasgo del titular del derecho. Sin embargo, si es la avaricia de los azules lo que justifica la inmunidad de los rojos a la manumisión, entonces tenemos que es un rasgo del sujeto incompetente - y no del inmune- el que justifica la restricción, con lo que, de acuerdo a la teoría de la restricción justificada, no habría derecho alguno. 
me parece correcto hablar de un derecho de los rojos a no ser liberados por los azules (pese a que los rojos son titulares de una inmunidad correlativa de una incompetencia restrictiva de lo que los azules pueden hacer). El hecho de que RAINBOLT señale al deseo de los azules de servirse de los rojos como la clave del derecho de aquellos sobre estos, me parece significativo, pues muestra como, a la hora de identificar derechos, tiende a reformular el proceso justificatorio para variar la asignación a la que lleva su propia teoría. Lo que RAINBOLT termina presuponiendo es que las restricciones de unos implican derechos de otros cuando son beneficiosas para estos ${ }^{38}$ ya que ante un derecho que necesariamente sea perjudicial o que no sea expresión del valor de sus titulares, tendemos a reformular el proceso justificatorio para alcanzar un resultado distinto sólido y no superfluo. Esa discriminación entre las restricciones que benefician y las que no es coherente con ciertas consideraciones que aparecen al final del libro de RAINBOLT ${ }^{39}$, donde se afirma que creer que existen restricciones que se justifican por ciertos rasgos de las personas es una forma de reconocimiento del respeto hacia esas personas. Estas afirmaciones son realmente razonables, pero no se corresponden con la definición canónica de la teoría que permite construir derechos a partir de rasgos que son un signo de la peor consideración hacia la persona de su titular. La definición del concepto de los derechos de la teoría de la restricción justificada es, por tanto, demasiado amplia en su referencia, ya que, al no señalar límite alguno a los rasgos fundantes de las restricciones, más allá del de ser rasgos de una persona y no del mundo, considera rasgos adecuados para fundar derechos a aquellos que no son expresivos de valor o signo de respeto alguno hacia su portador, sino de todo lo contrario. Sin embargo, como hemos visto, cuando la restricción de uno es signo de respeto por la persona de otro hablamos de derecho de éste y cuando no es así, como ocurre con el derecho a no ser liberado, no hay derecho alguno aunque sea el reflejo de una restricción ajena.

\section{TEORÍAS COMPLEJAS}

\subsection{La teoría de diversidad de funciones de L. WENAR}

La primera teoría que presupone diversidad interna en la referencia de los derechos y que, por tanto, no apuesta por un concepto unitario, es la teoría de la diversidad de funciones de L. Wenar. Wenar (2005, p. 224) afirma que HoHFELD es un buen punto de partida para elaborar una teoría de los derechos, aunque finalmente no sigue el esquema hohfeldiano de modo fiel sino que lo reelabora y complementa funcionalmente para construir el concepto de los derechos teniendo en cuenta lo que estos significan para su titular. A juicio de WENAR, por tanto, a la hora de identificar y definir un derecho, lo relevante es su función, es decir, lo que el derecho hace por su titular. WENAR refiere seis funciones definitorias de los derechos: exención, discrecionalidad, autoridad, protección, provisión y ejecución, que identifica inductivamente tras analizar diversos ejemplos de situaciones normalmente identificadas como casos

\footnotetext{
38 Así, por cierto, la teoría de la restricción justificada se aproxima a una versión justificatoria de la teoría del interés.

39 RAINBOLT, 2006, pp. 243-244.
} 
de derecho ${ }^{40}$. El catálogo que resulta tras considerar las seis funciones referidas sería el siguiente:

- El primer caso se da cuando decimos que alguien tiene derecho a hacer algo en el sentido de que no tiene el deber de no hacerlo. A esta instancia se la denomina comúnmente libertad, privilegio o licencia. WENAR señala que este tipo de libertad simple cumple la función de eximir a su titular de un deber general; en ese sentido, confiere una exención (exemption) (WENAR, 2005, p. 225).

- Uno puede tener también un derecho cuando es titular de dos libertades referidas respectivamente a la realización de un acto y a su omisión. En este caso, se tiene un derecho cuya función es la de conferir al titular discreción (discretion) o capacidad de elección referida a una acción específica (WENAR, 2005, p. 227). A estos derechos, de enorme importancia práctica, WENAR los llama paired privileges, aunque una denominación equivalente y más extendida es la de libertades bilaterales.

- Respecto de las situaciones en las que el Derecho se refiere a una acción de otro y aparece vinculado a su deber de llevar a cabo esa acción, son compatibles con las siguientes funciones: la protección (protection) frente al daño o frente a medidas paternalistas, la provisión (provision) en caso de necesidad o la ejecución (performance), por parte del obligado, de alguna acción específica acordada, compensatoria o procedente legal o consuetudinariamente (WENAR, 2005, p. 229).

- No solo tenemos libertades para hacer algo o derechos a que otros hagan algo, también existen derechos a cambiar libertades y derechos y derechos a que nuestros derechos y libertades no sean cambiados (WENAR, 2005, pp. 231-232). En el primer caso hablaremos de derechos de autoridad (authority) para alterar - crear, suspender o suprimir-, de acuerdo a un sistema de reglas dado, una situación normativa propia o de otro. Este tipo de instancias se corresponden con las normalmente denominadas «potestades» (WENAR, 2005, p. 231). Las potestades, como las libertades, pueden ser simples o compuestas: puedo estar habilitado para ejercer cierta autoridad no discrecional que tenga como efecto la modificación de alguna posición normativa subordinada - el caso del juez obligado a dictar sentencia al término del juicio es el paradigma - o bien tener una potestad que me otorgue cierta autoridad discrecional que me permite modificar alguna otra posición subordinada o no hacerlo. Como vemos, en el segundo caso la autoridad se combina con la discrecionalidad, es decir, que un derecho que consista en una pareja de potestades es a la vez un derecho de autoridad y de discrecionalidad, es decir, que refleja las dos funciones.

- Mención especial requieren las inmunidades que aparecen cuando una persona no tiene potestad para alterar ciertas relaciones normativas conforme a un sistema de reglas. Las inmunidades protegen a sus titulares frente a modificaciones en su marco o

40 HaYward (2006, p. 7) ha cuestionado esta selección de las funciones de los derechos por carecer de fundamento ya que lo que las relaciones hohfeldianas hacen por sus titulares iría más allá de las seis funciones especificadas, sin que WENAR justifique por qué selecciona, de entre todas las posibles, las seis que considera características de los derechos. Sin embargo, a mi juicio, la crítica es injustificada pues parte de la idea de que WENAR identifica las funciones tras analizar lo que las relaciones hohfeldianas hacen por sus titulares, cuando la identificación de WENAR se realiza tras observar lo que las situaciones que normalmente identificamos como derechos hacen por sus titulares. Como el propio WENAR (2005, p. 235) afirma, se llega a la conclusión de que los derechos cumplen esas funciones «examinando ejemplos de derechos». 
estatuto normativo; son, por ese motivo, derechos de protección (protection) frente al daño o al paternalismo (WENAR, 2005, p. 232).

En línea con lo afirmado en este trabajo, WenAR (2005, p. 237) sostiene que las teorías de la voluntad y del interés son single-function Theories of Rights, es decir, teorías que atribuyen a los derechos una única función, con lo que todos los derechos tendrían que manifestarla y nada que no lo hiciera podría ser tenido por un caso de derecho. Los teóricos de la voluntad, al definir los derechos por atribuir a su titular un poder discrecional sobre un deber de otro, sólo consideran derechos a aquellos que cumplen con la función de otorgar discrecionalidad y dejan fuera del universo de posibles titulares de derechos a todos aquellos privados de esa capacidad de ejercicio (WENAR, 2005, p. 239). Los teóricos del interés, por su parte, al asignar a los derechos la única función de promover los intereses o el bienestar de sus titulares, se ven en la imposibilidad de dar cuenta de figuras como los derechos que benefician a un tercero distinto de su titular o encuentran dificultades para explicar por qué en muchos casos el elemento discrecional es central en el sentido de que se reconoce a sus titulares el derecho a renunciar a lo que manifiestamente es de su interés. En consecuencia, según WENAR (2005, p. 223), tanto la teoría de la voluntad como la teoría del interés son demasiado estrechas, puesto que la función definitoria asignada por cada una de estas teorías a los derechos no llega a expresarse con claridad en todos y cada uno de sus casos. Igual que es fácil encontrar derechos que no definen un campo de libertad de su titular, también existen derechos cuya función no es promover los intereses de su titular, lo que coloca a ambas teorías ante un problema difícilmente solucionable. Adicionalmente, tampoco parece que el interés del titular juegue ningún papel relevante en el caso de la definición de los derechos vinculados a roles o funciones institucionales, como por ejemplo los derechos de los jueces o de los legisladores, cuyo ejercicio no es en interés propio, sino en interés de otros (WENAR, 2005, p. 241).

WENAR (2005, p. 246) se muestra, por estos motivos, partidario de una teoría de la diversidad de funciones según la cual cualquier relación o combinación de relaciones hohfeldianas será definida como un derecho siempre que lleve a cabo alguna o algunas de las seis funciones típicas de los derechos. Esta teoría es superior, afirma WENAR (2005, p. 247), a las teorías del interés y la voluntad, sin ser una simple superposición o concatenación de aquéllas, pues los casos excluidos por cada una de las teorías simples se verían contemplados por la teoría de la diversidad de funciones y además los derechos no contemplados ni por una ni por la otra ${ }^{41}$ quedarían también claramente identificados y definidos como tales ${ }^{42}$. Finalmente la teoría tendría la virtud adicional

${ }^{41}$ WENAR refiere como ejemplos la libertad bilateral de un individuo de contener el ataque a un tercero, el derecho del padre de castigar a un hijo desobediente o los derechos vinculados al ejercicio de funciones y roles públicos. Una lectura alternativa en clave de la teoría del interés en KRAMER y STEINER (2007, pp. 290292).

42 KRAMER y STEINER $(2007$, p. 294) afirman que, aunque WenAR pretende superar la teoría de la voluntad o del interés, finalmente lo que elabora es una versión de la teoría del interés en la medida en que las seis funciones definitorias de los derechos que refiere suponen usualmente un beneficio para los titulares del consiguiente derecho. Es decir, que cada una de esas funciones define apoderamientos o títulos que pertenecen a las clase de factores que normalmente promueven los intereses de los seres humanos, las colectividades o las especies no-humanas que pudieran ser tenidas como portadoras de algún tipo de interés. 
de incurrir en los posicionamientos normativos en los que habrían incurrido las teorías de la voluntad y del interés ${ }^{43}$.

Desde un punto de vista más teórico, la teoría de la diversidad de funciones vendría a reconocer o presuponer que los derechos no tienen una única utilidad para sus titulares, que pueden servirles de distintas formas y que cumplen diversas funciones en nuestras vidas: algunos nos protegen del daño incluso restringiendo nuestra autonomía, otros nos otorgan discreción para decidir en diversos sentidos y otros cumplen una función completamente distinta. La teoría de la diversidad de funciones encaja mejor, concluye WENAR (2005, p. 249), con nuestro entendimiento ordinario sobre los derechos y es más adecuada a la comprensión habitual de los derechos, al sentido común de lo que son y de lo que representan en la vida social o en la realidad jurídica. También expresaría respeto por la historia, a lo largo de la cual se ha ido generando el concepto de los derechos pues, pese al atractivo de las teorías simples y de la búsqueda teórica de la unidad o de lo general, el sentido último del concepto de los derechos no podría ser simplificado y ni su significado final el resultado de destilar ningún principio normativo, sino una suma o una mezcla resultado de principios, valores y funciones diversas que han venido atribuyéndose al fenómeno a lo largo de la historia, desde sus orígenes hasta la actualidad.

\subsection{La teoría híbrida de G. SREENIVASAN}

G. SREENIVASAN (2005) ha propuesto recientemente una teoría híbrida de la teoría del interés y la voluntad ante la constatación de que carecemos tanto de una solución apropiada al debate sobre si es el interés o la voluntad lo que define a los derechos, como de una teoría que ofrezca una interpretación apropiada de la naturaleza de la relación del titular del derecho con la obligación vinculada a los derechos. Para SREENIVASAN el elemento determinante de la relación está relacionado con la justificación de la medida de control que alguien ejerce sobre los deberes de otro ${ }^{44}$. Según la teoría híbrida en su versión cruda o simple (SREENIVASAN, 2005, p. 267):

«Supuesto que $\mathrm{X}$ tiene un deber de $\rho, \mathrm{Y}$ tiene un derecho frente a $\mathrm{X}$ sólo en el caso de que:

o bien Y tiene el poder de renunciar a que X cumpla con su deber de $\rho$

o bien $\mathrm{Y}$ no tiene ese poder de renunciar a que $\mathrm{X}$ cumpla con su deber, pero (esto es, porque) la incompetencia de $\mathrm{Y}$ promueve a fin de cuentas los intereses de $\mathrm{Y}$ ».

Existe una versión más refinada o compleja de la teoría que SREENIVASAN (2005, pp. 269-271) propone tras analizar una serie de variables y circunstancias que pudieran afectar a la situación típica. La versión refinada o compleja dice que:

«Supuesto que $\mathrm{X}$ está obligado a $\rho, \mathrm{Y}$ tiene un derecho a que $\mathrm{X}$ haga $\rho$ sólo en el caso de que la medida de control de $\mathrm{Y}$ (de $\mathrm{Z}$ en el caso de que $\mathrm{Z}$ actúe en lugar de $\mathrm{Y}$ ) sobre un

43 HAYWARD (2006, p. 7), sin embargo, considera que tampoco la teoría de WENAR es neutral ya que la selección de las funciones definitorias de los derechos también estaría condicionada normativamente aunque de un modo subrepticio.

44 KRAMER y STEINER (2007, p. 299) reprochan a SREENIVASAN que no haya perfilado con mayor precisión el concepto de justificación, pese a ser central en su teoría. 
deber de $\mathrm{X}$ de hacer $\rho$ coincide (por diseño) con la medida de control que promueve, a fin de cuentas, los intereses de $Y »$.

Esta es una teoría con dos entradas fruto de la yuxtaposición de las tesis de la voluntad y el interés. Por ese motivo, los problemas que ambas teorías suscitan se pueden solucionar recurriendo a la entrada correspondiente de la teoría híbrida, cuyo carácter disyuntivo le permite eludir las críticas respectivas. Por ejemplo, recordemos como la teoría de la voluntad encontraba problemas para dar cuenta de algunos derechos de naturaleza inalienable. Estos, desde el punto de vista de la teoría híbrida de SREENIVASAN, se explicarían por su segunda entrada: la incompetencia para renunciar al derecho o, mejor, a la realización de la obligación correlativa, es la forma de asegurar los intereses del titular que los derechos protegen (SREENIVASAN, 2005, p. 267). La teoría híbrida también permite solucionar los principales problemas que plantean las teorías del interés como, por ejemplo, el caso de los terceros beneficiarios no titulares de derechos. Recordemos que este problema se plantea cuando la teoría del interés, al definir el derecho en función del interés, identifica como titular a todo posible beneficiario del cumplimiento de la obligación. Desde el punto de vista de la teoría híbrida, sin embargo, la titularidad del derecho se explicaría por referencia a la primera entrada de la definición, con lo que el titular en cuestión sería aquél a quien se ha formulado el compromiso y que tiene capacidad para extinguir la obligación correlativa.

También las obligaciones implícitas a las normas penales dejarían de ser un problema en el contexto de esta teoría híbrida. Según algunos críticos de la teoría de la voluntad, la incapacidad de sus partidarios para dar cuenta de los derechos protegidos por las normas de derecho penal es un argumento contra esta teoría; en paralelo, el hecho de que de la teoría del interés se siga que los individuos protegidos por las normas de Derecho penal tienen derechos es, desde posiciones críticas a la teoría del interés, un argumento contra esa teoría, puesto que este tipo de normas no generarían derecho alguno de los individuos protegidos. Desde el punto de vista de la teoría híbrida, la respuesta al problema depende de la razón por la que se priva u otorga a alguien control sobre el cumplimiento o la ejecución de las normas penales. Cuando se priva a los particulares de control para otorgarlo a algún fiscal o autoridad acusadora porque de ese modo se protegen mejor los intereses de los individuos protegidos, entonces diremos que éstos tienen derechos según la segunda entrada de la regla de la teoría. Si la inhabilitación de los individuos y la consiguiente asignación del control a la autoridad se justifica por razones de tipo público, ajenas a los intereses de los protegidos por las normas, entonces éstos no serán titulares de derecho alguno correlativo a las prohibiciones y deberes establecidos por las normas penales.

La teoría híbrida, por su sofisticación y por su rigor, parece merecedora de la mayor atención teórica. Su elegante formulación es expresiva del modo en que se han acumulado algunas generaciones de derechos y resuelve de un modo muy sugerente los problemas teóricos planteados a la hora de contrastar la función de ciertas categorías de derechos ${ }^{45}$. En mi opinión la forma en que la teoría híbrida intenta superar el debate entre voluntad o interés, no le permite remontarlo de modo definitivo. La teoría híbrida no deja de ser un intento de integrar ambas teorías bajo una fórmula disyuntiva:

${ }^{45}$ Me refiero a derechos de libertad y a derechos sociales. 
o voluntad o intereses. Por este motivo, la propuesta de SREENIVASAN no puede librarse de algunas de las críticas u objeciones que se plantean entre sí los respectivos partidarios de cada uno de los dos polos clásicos en disputa. Para los partidarios del interés, una versión expandida de esta teoría, en la que el concepto de interés se estira hasta el punto de abarcar la libertad para decidir, siempre será preferible ${ }^{46}$. Los partidarios de la teoría de la voluntad, por su parte, no han tardado en reprochar a SREENIVASAN que el grado de control o disposición sobre el deber correlativo del titular del derecho bien pudiera ser ninguno si eso es lo que favorece sus intereses en conjunto, con lo que la teoría híbrida queda expuesta a todas las críticas referidas al carácter paternalista que los teóricos de la voluntad ya habían dirigido a la teoría del interés ${ }^{47}$.

\section{CONCLUSIONES}

Al margen de críticas a cuestiones más o menos puntuales, el factor determinante de la superioridad teórica de estas dos últimas propuestas — las de WENAR y SREENIVASAN - reposa sobre un presupuesto de todo el análisis: la homogeneidad de la referencia de los derechos y, por tanto, la posibilidad de elaborar o no una definición simple que abarque todos y cada uno de los casos del concepto. Antes de apostar por una teoría simple o compleja, hay que determinar si la referencia del concepto de los derechos es internamente homogénea o si, por el contrario, adolece de algún grado de complejidad, de donde se seguiría que no hay un único tipo de derechos, ni, por lo tanto, es posible una teoría que defina a todos y cada uno de sus casos posibles en función de un único rasgo o de una lista cerrada de rasgos.

Si la referencia es efectivamente compleja, las teorías simples sólo podrían abarcarla ampliando el sentido del rasgo o rasgos definitorios para poder localizarlos en todos y cada uno de los casos del concepto. Esto es lo que, como hemos visto, hacía RAINBOLT al definir como derechos a los correlativos de las restricciones fundadas en algún - lo que es tanto como decir «cualquier»— rasgo de su titular, con los efectos descritos anteriormente ${ }^{48}$. También es lo que hacen algunas versiones de la teoría del interés ${ }^{49}$ para abarcar conceptualmente aquellos derechos en los que lo primordial es

46 Esa es la tesis de fondo de KRAMER y STEINER quienes, pese a la crítica, llegan a manifestar que estamos ante una genuina alternativa a las teorías de la voluntad y del interés (KRAMER y STEINER, 2007, p. 308). Estos mismos autores plantean alguna corrección de interés a la teoría híbrida para evitar que esta teoría pudiera implicar el reconocimiento de un derecho a los interesados en no disponer de control alguno sobre relaciones normativas de terceros. Es el caso de un sujeto ajeno a una relación acreedor-deudor pero interesado en no ser competente para eximir al deudor del cumplimiento de su deber para evitar, por ejemplo, posibles represalias que el acreedor podría plantearle en caso de que disfrutara de ese control. Puesto que al carecer del control sobre el pago, se favorecen los intereses de ese tercero extraño a la relación principal, deberíamos concluir, de acuerdo a la teoría híbrida, afirmando un derecho a favor de este tercer sujeto, algo que, sin embargo, no parece tener mucho sentido (KRAMER y STEINER, 2007, p. 309).

47 SPECTOR (2007, pp. 296-298), por ejemplo, ha señalado que la teoría híbrida es una forma de legitimar el paternalismo iliberal y que está tan expuesta a las objeciones relativas a la aceptación del cálculo utilitario de derechos como lo estaba la teoría del interés. Otro análisis similar de la teoría híbrida de este mismo autor puede encontrarse en SPECTOR, 2005, pp. 799 y ss.

${ }_{48}$ Un problema añadido de este tipo de soluciones y teorías es que parecen presuponer que el lenguaje del derecho obedece a una gramática regular y precisa, sujeta a leyes constantes y sin excepciones, y reflejo de una realidad que los usos sociales habrían pervertido u ocultado.

49 Este es el caso, en mi opinión, de la versión de KRAMER y STEINER (2007). 
la capacidad de su titular para elegir y no el carácter benéfico de lo elegido. Recordemos que este último tipo de derechos terminan siendo explicados como una manifestación del interés de los individuos a decidir autónomamente sobre ciertas cuestiones. No obstante, considerar que el derecho a suicidarse, a practicar el sexo sin protección o a no someterse a una operación quirúrgica que le salvará a uno la vida o le evitará terribles padecimientos son una expresión de los intereses de su titular, es destructivo de la propia noción de interés, pues obliga a considerar como tal el interés en perjudicarse y define como bienestar a todo aquello que beneficia a los individuos más algunas otras cosas que claramente los perjudican (!). Es teóricamente más plausible considerar que algunos derechos se definen y encuentran su fundamento en la autonomía de los individuos, al margen del carácter benéfico o no de su ejercicio, y que otros derechos, por el contrario, hacen lo propio con los intereses de sus titulares.

Esta conclusión anticipa la respuesta a la cuestión a la que me refería al inicio de este apartado: ¿es la referencia de los derechos homogénea o, por el contrario, los casos de derecho no responden a un único patrón o constante? En mi opinión, la solución a la pregunta anterior es clara: la referencia del concepto de los derechos no sólo no es homogénea, sino que está afectada por un elevado grado de heterogeneidad e incluye elementos diversos e irreductibles a un patrón común, lo que hace inviable una definición unitaria.

Basta analizar someramente el estado actual de los derechos y, en especial, su proliferación, para ilustrar esa conclusión. WELLMAN (1997, p. 2) ha dedicado especial atención a la proliferación de los derechos, fenómeno que se habría producido en tres planos: proliferación de derechos morales, proliferación de derechos legales y, por último, la proliferación del lenguaje de los derechos en el discurso político. SUMNER (1987, p. 1), por su parte, habla de escalada en la retórica de los derechos tanto en el plano del discurso y del debate político como en los textos llamados a funcionar como textos canónicos de los derechos. Termina, por cierto, adoptando cierto tono catastrofista al señalar que la escalada en la retórica de los derechos, como la escalada armamentística, está fuera de control. Las consecuencias de esa proliferación no son siempre necesariamente favorables. La expansión — generalmente mediante cláusulas y fórmulas vagas y retóricas - plantea, en primer lugar, un problema práctico, pues compromete la fuerza vinculante general de los derechos, es decir, su carácter indiscutible o no-excepcionable tanto en el ámbito político como en el específicamente jurídico ${ }^{50}$. Pero también,

50 De un modo muy expresivo, LOMANSKY (2000, p. 104) también expresa sus reservas a la confusión entre derechos y virtudes, ideales, santidad o heroísmo moral. Un contexto regido por los derechos, afirma, no es lo mejor que podemos concebir ni lo máximo a lo que podemos aspirar, pues lo que nos proporcionan los derechos son cautelas y salvaguardias frente a lo peor, pero en modo alguno garantizan lo mejor. Aunque todos podríamos y deberíamos hacer más por los demás que limitarnos a respetar sus derechos, los derechos marcarían el mínimo necesario e infranqueable. La contrapartida a estas pocas exigencias es su máximo peso pues los derechos exigen de nosotros unas pautas de comportamiento reducidas o mínimas hacia los otros, pero las exigen hacia todos y en cualquier circunstancia, incluso respecto de aquellos que nos resultan repudiables $\mathrm{u}$ odiosos. Respetar los derechos de alguien no debe de suponernos ningún acto de heroicidad, sino un sustrato básico mínimo. Una cosa sería recomendar el heroísmo moral y el compromiso ciego o la entrega absoluta hacia los demás y otra cosa exigirlos. Las declaraciones maximalistas de los derechos se desacreditan a sí mismas en la medida en que exigirían un nivel de excelencia, compromiso y sacrificio moral de todos y cada uno de aquellos a los que se dirigen muy elevado y que va mucho más allá de lo que, en general, todos y cada uno de nosotros estaríamos dispuestos a satisfacer. 
en segundo lugar, desdibuja los perfiles del concepto mismo de los derechos. Así hay quien advierte que hoy en día se llama «derecho» a cualquier cosa de valor (RAZ, 1986, p. 165), lo que disipa la consistencia interna de la referencia de los derechos y supone un reto para las teorías que los explican ${ }^{51}$.

El contexto dibujado por todas esas opiniones hace prácticamente inviable la pretensión de ofrecer una definición simple de los derechos ${ }^{52}$; los lamentos de quienes llaman la atención sobre el fenómeno de la proliferación y sus consecuencias prácticas y teóricas, pudieran entenderse como el intento de preservar una esencia que los derechos parecen no tener. Por su parte, la alternativa planteada, esto es, el escepticismo respecto de la posibilidad de encontrar constantes teóricas en la definición, podría ser considerada una suerte de inquietante ${ }^{53}$ renuncio teórico. Lamentablemente, teniendo en cuenta la evolución legal y doctrinal de los derechos y su actual estado, es difícil que cualquier teoría alcance los estándares de éxito teórico deseables, si éxito se define en virtud de parámetros como la generalidad de la definición o la localización de rasgos constantes y definitivos ${ }^{54}$. Toda pretensión de esta naturaleza, en mi opinión, se verá forzada a formular un concepto que no podrá librarse de excepciones flagrantes o inclusiones absurdas. Opino que una teoría y un concepto realista de los derechos deberían de tomar en consideración todas esas circunstancias, incluyendo especialmente los cambios históricos que han afectado en el pasado al concepto de los derechos e incluso anticipar posibles vías de evolución inmediata ${ }^{55}$.

51 RAZ (1986, p. 63) afirma también que cuando uno aborda conceptos como el de los derechos, que están fuertemente vinculados a tradiciones y visiones políticas contrapuestas de nuestra propia tradición y nuestra cultura, es inevitable ofrecer una lectura un tanto parcial de su uso que pretende favorecer una visión frente a otras contrapuestas.

52 La teoría de la voluntad es quizás el mejor exponente tanto de esa pretensión y de la propia suposición de una imagen unitaria y coherente de los derechos. Su evolución es también la mejor muestra del fracaso y de la esterilidad de ese empeño. Lo explica SPECTOR (2007, p. 292) para quien la teoría de la voluntad es simple y homogénea, como lo era, allá por los siglos XVII y XVIII, el fenómeno del que daba cuenta. Sin embargo, aquella imagen armoniosa se vino abajo a mediados del siglo XIX, en el momento en que los valores y principios vinculados con la autonomía moral fueron sustituidos por un nuevo paradigma centrado en intereses y objetivos sociales. Entonces los derechos dejaron de ser concebidos como integrantes del estatus de sujetos autónomos y pasaron a ser concebidos como instrumentos para promover intereses de los individuos o para alcanzar ciertos objetivos sociales. La teoría del interés, inspirada por la nueva sensibilidad, explicó esos cambios, aunque, dicho sea de paso, no termina de casar del todo con los derechos basados en el valor de la autonomía y que persisten de la etapa anterior.

53 Es así como ELEFTHERIADIS (2008, p. 19) califica a la teoría de WENAR ya que, a su juicio, esperamos que una teoría de los derechos sea capaz de desvelar una idea sobre los mismos que se refiera a algo coherente o a algún tipo de generalización iluminadora. El propio WENAR habría reconocido ese problema, pero insiste que es imposible satisfacer la pretensión de unidad, porque cuando el teórico la aplica al fenómeno y al concepto de los derechos, encuentra con que el objeto manifiesta una irreducible complejidad y que los derechos han sido instrumentalizados con fines diversos en el razonamiento legal o moral.

54 Quizás el conocido debate entre CRANSTON y RAPHAEL a propósito de la naturaleza de los derechos sociales y de su relación con los derechos y libertades civiles, nos ofrezca un buen ejemplo del modo en que ha evolucionado la noción de los derechos. Los argumentos de CRANSTON en ese debate, que presuponían la exclusión de los derechos sociales y económicos del ámbito de referencia del concepto de los derechos, no parecen hoy asumibles, porque nuestra visión del concepto ha variado sustancialmente. Sin embargo, en su día, probablemente por estar más próximos a un entendimiento decimonónico o en clave liberal de los derechos aún extendido entonces, sí tuvieron cierto peso. El debate en cuestión puede consultarse en WELLMAN, 2002, pp. $1-25$.

55 Mientras que llega el futuro, algunas conclusiones sí parecen posibles en el contexto actual y cuestionarían, por ejemplo, la pretensión de ofrecer un molde o marco unitario de las formas de reconocimiento o las garantías de los derechos. Por ejemplo, la tan reclamada asimilación de las garantías de los derechos sociales a 
Por todos esos motivos considero que las tesis de Wenar y de SREENIVASAN son más adecuadas que las otras alternativas estudiadas. Sin embargo, no hay que descartar que su adecuación sea finalmente superada y que estas propuestas terminen siendo finalmente desbordadas. Consideremos, en prospectiva, el fenómeno de la objetivización del contenido de los derechos o la expansión de los llamados «derechos de justicia» ${ }^{56}$ : si como consecuencia de una evolución en esa dirección, los derechos se segregaran tanto de la voluntad como de los intereses de sus titulares y pasaran a ser concebidos como puras exigencias de justicia que habrían de realizarse al margen de cualquier consideración subjetiva ${ }^{57}$, entonces referencias teóricas como las que definen a los derechos por lo que hacen por sus titulares o por favorecer o su voluntad o sus intereses, podrían ser vistas como obsoletas o pasar a ser estrechas. La posibilidad de fracaso teórico no es el único efecto que la evolución y la proliferación de los derechos plantean; hay que considerar también la provisionalidad de cualquier planteamiento aparentemente exitoso en la actualidad.

\section{BIBLIOGRAFÍA}

Bentham, J., 1782: On Laws in General, London, The Athlone Press, 1970.

CÁCeres, E.; Flores, I.; SAldaña, J., y Villanueva, E., 2005: Problemas contemporáneos de la filosofía del Derecho, México, UNAM.

CARRIÓ, G., 1968: «Nota preliminar», en HOHFELD, 1919.

DABIN, J., 1952: Le droit subjectif, Dalloz, Paris (hay traducción al español de F. J. OssET por donde se cita: El derecho subjetivo, Madrid, Editorial Revista de Derecho Privado, 1955. Reeditado más recientemente El derecho subjetivo, Granada, Comares, 2006).

Eleftheriadis, P., 2008: Legal Rights, Oxford, Oxford University Press.

GRIMm, D., 1991: Die Zukunft der Verfassung, Frankfurt, Suhrkamp (citado por la traducción al español de R. SANZ y J. L. MuÑOZ DE BAENA, Constitución y derechos fundamentales, Madrid, Trotta, 2006).

HALPIN, A., 2003: «Fundamental Legal Relations Reconsidered», en Canadian Journal of Law and Jurisprudence (vol. XVI, núm. 1, enero).

HART, H. L. A., 1961: The Concept of Law, Oxford Clarendon Press (citado por la traducción al español de G. CarRió, El concepto de derecho, México, Fontamara, 1991).

- 1982: Essays on Bentham, Oxford, Clarendon Press.

HAYWARD, T., 2006: «On the Nature of Rights: A Reply to Wenar» (1 de febrero de 2006). Disponible en SSRN: http://ssrn.com/abstract=893340.

Hierro SÁnCHEZ-PesCADOR, L., 2000: «Conceptos jurídicos fundamentales (I): de las modalidades deónticas a los conceptos de derecho y deber», en Revista Jurídica de la Universidad Autónoma de Madrid, núm. 3.

Hohfeld, W. N., 1919: Fundamental Legal Conceptions as Applied in Judicial Reasoning, Greenwood Press, Westport, 1978 (hay traducción al español, con «Nota Prelimilar», de G. CARRió, Conceptos jurídicos fundamentales, México, Fontamara, 1991).

los liberales generalmente se sustenta en una supuesta afinidad conceptual entre ambas categorías, que, como se ha visto a lo largo de este análisis, no parece viable o no parece que vaya más allá de una simple coincidencia terminológica.

56 GRIMM (1991, p. 155) y ZAGREBESLKY (1992, p. 75).

57 Habríamos terminado entonces teniendo derecho a lo que es justo, al margen de que lo deseemos y al margen de que nos beneficie o no. 
Kavanagh, A., y OberdieK, J., 2008: Arguing About Law, London-New York, Routledge.

Kramer, M., 1998: «Rights without Trimmings», en Kramer, Simmonds y STEInER.

Kramer, M., y SteIneR, G., 2007: «Theories of Rights: Is There a Third-Way?», en Oxford Journal of Legal Studies, vol. 27, núm. 2.

Kramer, M.; Simmonds, N. E., y Steiner, G., 1998: A Debate over Rights, Oxford, Clarendon Press.

LAPORTA, F., 1987: «Sobre el concepto de derechos humanos», en Doxa, 4.

LOMANSKY, L., 2000: «Liberty and Welfare Goods: Reflections on Clashing Liberalism», en The Journal of Ethics, 4.

MACCORMiCK, N., 1976: «Children's rights: a test-case for theories of right», en Archiv für Rechts- und Sozialphilosopbie, 67.

— 2008: «Rights in Legislation», en KaVANAGH y OBERDIEK.

Rainbolt, G., 2006: The Concepts of Rights, Kluwer, Dordrecht.

RAz, J., 1986: The Morality of Freedom, Oxford, Clarendon Press.

— 2008: «Rights and Individual Well-being», en KAVANAGH y OBERDIEK.

Ross, A., 1958: On Law and Justice (hay traducción de G. R. CARRIÓ, por donde se cita: Sobre el Derecho y la justicia, Buenos Aires, Eudeba, 1963).

Simmonds, N. E., 1998: en KRAmer, SimmOndS y STEINER.

SREenivasAn, G., 2005: «A Hybrid Theory of Claim-Rights», en Oxford Journal of Legal Studies, vol. 25, núm. 2 (también en CÁCERES, Flores, SALDAÑa y VillanueVA, 2005).

SpeCTOR, H., 2005: «Comment on professor Gopal Sreenivasan's Hybrid Theory of ClaimRirghts», en CÁCERES, Flores, SALDAÑa y VillanueVa.

— 2007: «Is the Will Theory Superseded by the Hybrid Theory?», en VillanuEva.

STEINER, H., 1994: An Essay on Rights, Oxford, Blackwell Publishers.

Sumner, L. W., 1987: The Moral Foundations of Rights, Oxford, Clarendom Press.

Villanueva, E., 2007: Law: Metaphysics, Meaning and Objectivity, Amsterdam-New York, Rodopi Philosophical Studies.

Waldron, J., 1984: Theories of Rights, Oxford, Oxford University Press.

Wellman, C., 1997: An Approach to Rights, Dordrecht, Kluwer.

- 2002: Rights and Duties, vol. 5 Welfare Rights and Duties of Charity, New York, Routledge.

Wenar, L., 2005: «The Nature of Rights», en Philosophy and Public Affairs, 33, 3.

Zagrebelsky, G., 1992: Il diritto mitte, Torino, Einaudi (citado por la traducción de M. GASCón Abellán, El derecho dúctil, Madrid, Trotta, 1995). 